\title{
Coelestis Musica. La solución de Honorio de Autum
}

\author{
Coelestis Music. The Honorius of Autun solution
}

\author{
Laura Carolina Duran \\ Instituto de Filología Clásica, Facultad de Filosofía y Letras, Universidad de Buenos Aires, Argentina. \\ (lcarolinaduran@gmail.com)
}

Resumen: La teoría de la música de las esferas formula una organización armónico-musical del universo. Iniciada en la Antigüedad clásica se mantuvo vigente durante la Edad Media, constituyendo un eje central en la transmisión del pensamiento pitagórico-platónico. Se reelaboró a lo largo del tiempo. En esas nuevas versiones se intentaba responder a las tempranas críticas aristotélicas. En este trabajo proponemos una reconstrucción de los argumentos de Honorio de Autun al respecto, dado que resultan de capital importancia, en tanto procuran establecer vínculos entre la teoría de la música de las esferas y la concepción del denominado quinto elemento: el éter. Con estas articulaciones Honorio intentaba salvar la doctrina de la música cósmica de las observaciones y hasta rechazos, basadas en la inaudibilidad de esta música de los astros.

\section{Palabras clave}

Música de las esferas, éter, Edad Media, Honorio de Autun.

\begin{abstract}
The theory of the music of the spheres formulates a harmonic-musical organization of the universe. Initiated in Classical Antiquity, it remained in force during the Middle Ages, constituting a central axis in the transmission of Pythagorean-Platonic thought. It was reelected over time. In these new versions an attempt was made to respond to the early Aristotelian criticism. In this paper we propose a reconstruction of the arguments of Honorius of Autun in this regard, given that they are of capital importance, while trying to establish links between the theory of the music of the spheres and the conception of the so-called fifth element: the ether. With these articulations Honorio tried to save the doctrine of the cosmic music of the observations and even rejections, based on the inaudibility of this music of the stars.
\end{abstract}

Keywords: Music of the spheres, ether, Middle Ages, Honorius of Autun. 


\section{Introducción}

A lo largo de la Antigüedad, Tardoantigüedad y Medioevo la reflexión sobre la música tuvo un sitio de gran relevancia. Las teorías antiguas, recopiladas por Agustín y Boecio, fueron transmitidas a la Edad Media.

La música era considerada una propiedad universal de las cosas, “decir música es decir armonía" escribió Hugo de San Víctor (Villalaz, 2011, p.43). Los medievales mantuvieron las principales tesis antiguas, en particular el principio pitagórico de que lo esencial en de la música era la proporción y el número. De modo que predominaba la interpretación teórica que provenía del pitagorismo, del Timeo platónico y de Boecio. La proporción no se entendía como producto del intelecto humano, por el contrario, la razón "el sentido interior del alma” (Luque Moreno- López Eisman, 2007, p.103), la captaba del mundo real. "La armonía se encuentra en todas las criaturas" (Eco, 2013, p.45; Tatarkiewicz, 1989, p.133)1.

Desde la Tardoantigüedad se dividía esta disciplina en: música del universo, música del hombre, y música de las obras humanas (Luque, 2009, pp.76-79). La musica mundana de Boecio fue la versión de la música de las esferas que circuló en el Medioevo. La idea fundamental era que los planetas, en su eterno girar, producían un acorde de sonidos armonioso. La teoría articulaba diversos estudios: aritmética, geometría, astronomía y música: las artes del quadrivium, propias de lo real. Honorio de Autun realizó ciertas consideraciones sobre esta música. Presentaremos algunas referencias del autor y su obra para luego revisar su versión de la música de los cielos.

\section{Honorio. El autor y su obra.}

Honorio fue un filósofo del siglo XII. Poco se conoce de su vida. Vivió ca.1080- 1153 (Gottsachall, 1992, p.8; Flint, 1982, pp.7-8). En De luminaribus Ecclesiae afirma vivir bajo el reinado de Enrique V (Migne, 1857-1866, CLXXII, col.10)². Alrededor de 1100 se instala en Inglaterra y estudia con Anselmo de Canterbury (Roth, 1939; Crouse, 1975). Fue sacerdote y maestro en Autun. Parece

\footnotetext{
${ }^{1}$ Consonantia habetur in omni creatura, la cita que toman Eco y Tatarkiewics pertenece a Otloh de San Emerano.

2 Enrique V gobernó el Sacro Imperio romano germánico, entre 1106 y 1125. Las traducciones son propias.
} 
buscar mantener cierto misterio sobre sí mismo según leemos en el Praefatio de Elucidarium: "pero mi nombre quise cubrir con silencio" (Migne, 1857-1866, CLXXII, col.1110)³.

De acuerdo con Flint (1972), tomó los hábitos en una abadía benedictina, cerca de Ratisbona. Su obra es variada y tuvo gran circulación en su época. La crítica en general acuerda que su trabajo se caracteriza por recopilaciones, a la vez que persigue un intento de ordenamiento y clarificación; sin pretender originalidad alguna. Su obra representa un enlace entre elementos del Renacimiento carolingio y las Escuelas del siglo XIII. J. P. Migne dedica el vol. CLXXII a la obra de Honorio ${ }^{4}$ que no está del todo completa; existe controversia al respecto ${ }^{5}$. Una visión general del autor, podría presentarlo como un asiduo estudioso de las más variadas disciplinas, en busca una síntesis del conocimiento. Los textos que tratan sobre la estructuración del mundo contienen apreciaciones sobre la música de las esferas.

\section{De imagine mundi.}

De imagine mundi y Elucidarium eran obras muy conocidas. Ambas constan de tres libros. El libro I de De imagine trata sobre el cosmos, la geografía del mundo, su composición (los elementos), los cuerpos celestes, los planetas y las estrellas. El segundo trata del "tiempo en el que estamos" y el tercero recurre al tiempo histórico y relata la progresión de las seis edades del mundo, hasta el Sacro Imperio Romano. Este tratado se diferencia del Elucidarium formalmente, una narrativa simple frente al diálogo; y el trato hacia las autoridades, mencionadas en Elucidarium, ausentes en De imagine ${ }^{6}$.

Flint (1982) identifica las fuentes: Plinio, Solino, Orosio, Macrobio, Isidoro, Marciano, Beda, Rabano Mauro, Helperico de Auxerre, Pseudo-Beda, Pseudo-Alcuino, las historias ortodoxas. Según la editora, la obra no tiene novedad ni en su contenido ni en los materiales (1982, p.13). Sin embargo, le reconoce ciertas sorpresas (1982, p.14): la forma en que Honorio utiliza las fuentes aun en capítulos muy breves suele haber más de una—, la elección de los materiales — que dan

\footnotetext{
3 Nomen autem meum ideo volui silentio contegi. Se consultó también la edición de Luff (1999). En otra obra, Sigillum Beatae Mariae realiza una afirmación muy parecida.

${ }^{4}$ La ordenación de la obra que reliza Migne es la siguiente: (a) Didascálicos e históricos: De philosophia Mundi, De solis affectibus, De imagine mundi, Summa totius de omnimoda historia, De scriptoribus ecclesiasticis: (b) Exegéticos: Hexaemeron, De decem plagis Aegipti spiritualiter, Expositio psalmorum selectorum, Quaestiones et responsiones in Proverbia, Expositio in Cantica Canticorum, Sigillum B. Mariae; (c) Litúrgicos: Gemma animae, Sacramentarium, Speculum Ecclesiae; (d) Dogmáticos y ascéticos: Elucidarium, Liber duodecim questionum, Libellus octuo quaestionum, Inevitabile sive Dialogus de libero arbitrio, Scala coeli Major, Scala coeli minor, De animae exsilio et patria, De vita claustrali, Eucharistion, Summa gloria de Apostolico et Augusto.

5 A este respecto los trabajos de Flint (1972, 1977, 1982) y Garrigues (1986) aportan adecuada información.

${ }^{6}$ Flint (1982). Si bien consignemos que ocasionalmente menciona a ciertos autores al mentar sus desarrollos, como Pitágoras, Platón, etc.
} 
relieve al propósito del tratado como un todo-. Flint conjetura que esto obedece a un objetivo político (1982, p.16). En la carta preliminar Honorio sostiene "pues en esto nada introduzco, sino lo que recomienda la tradición de los mayores" $\left(1982\right.$, p.49) ${ }^{7}$. Honorio deseaba que su tratado gozara de un atractivo general, como reconoce en el prefacio "para la instrucción de muchos, a quienes falta el suministro de libros" (1982, p.49).

\section{1. La música y los planetas.}

Dada la vinculación al Timeo platónico, recordemos que se ha atribuido a Honorio un Comentario sobre el Timeo (Calabrese, 2015). En ese texto se divide a la filosofía en práctica y teórica, subdividida esta última a su vez en teología, matemática y física. La matemática es el "quadrivium: la doctrina es más perfecta en el quadrivium que en las otras artes" (Calabrese, 2015, p. 2) ${ }^{8}$. Reitera la clasificación de la música de Boecio en instrumental, mundana y humana (Friedlin-Mathiesen 1994, I, 2, 187-188) ${ }^{9}$.

Encontramos una explicación detallada de la musica mundana en De imagine. Allí, luego de haber tratado sobre la geografía del mundo, los cuatro elementos y sus diversas expresiones incluida una descripción del infierno que será modelo para Dante-, pasa a estudiar los planetas. Establece que son siete y giran en sentido contrario al mundo, tal como hiciera Platón en Timeo y República X (Flint, 1982, p.76). Destina un breve capítulo a cada uno, continúa con las respectivas ubicaciones en relación con la esfera de las estrellas fijas, sus colores y sus sonidos. Honorio afirma en el apartado De sono illorum: "estos siete globos ruedan con armonía, y unos suavísimos acordes son producidos por su rotación" (Flint, 1982, p.79) ${ }^{10}$.

El capítulo siguiente se titula Coelestis musica. Aceptada la emisión de sonidos de los planetas, Honorio va a establecer la escala musical. Llamativamente propone dos escalas heterogéneas. El orden de los astros es el mismo que presenta Cicerón: la Tierra fija en el centro (no produce sonido alguno) luego la Luna, Mercurio, Venus, el Sol, Marte, Júpiter y Saturno. Entre los autores que dejaron escritos sobre la armonía cósmica suelen ocurrir variaciones en la posición de

\footnotetext{
7 Para las citas del texto latino seguimos la edición de Flint (1982), a la vez que se consultó la edición de Patrologia Latina de Migne (1857-1866, CLXXII)

8 Agreguemos que, cuando Honorio inicia realmente el comentario - luego de explicar el propósito de la obra platónica y analizar la versión de Calcidio- comienza por las primeras palabras: uno, dos, tres, y refiere que en los números está la máxima perfección pues estos números son los propios de los intervalos musicales.

${ }^{9}$ En el Comentario continúa señalando que la música instrumental, a su vez, comprende tres clases: mélica, métrica y rítmica. La mélica, por su parte, abarca tres tipos: diatónica, enarmónica y cromática.

10 Hi septem orbes cum dulcisona harmonia volvuntur, ac suavissimi concentus eorum circuitione efficiuntur. Destaquemos que los términos que utiliza Honorio son los mismos empleados por Cicerón en el Somnium, con mínimas diferencias, a la vez que se distancian en cierta medida de los utilizados por Boecio.
} 
algunos planetas ${ }^{11}$. Las dos escalas musicales son: por una parte, una que otorga una nota a cada planeta, siguiendo la escritura de Guido d’Arezzo (Luna A, Mercurio B, Venus C, Sol D, Marte E, Júpiter F, Saturno G, nuestra escala de La), y por otra, una reducción de las distancias interplanetarias de Plinio a una escala de siete tonos (T, s/t, s/t, T s/t, T, s/t, s/t, T s/t) — distancias referidas en el capítulo De mensura (Flint, 1982, p. 79)—12.

Esta doble escala musical planetaria responde a dos formas de conceptualización. De un lado, la atribución de una nota específica a cada planeta es una tradición iniciada por Nicómaco de Gerasa y continuada por Boecio, autores que entienden que la producción del sonido responde a movimientos, posiciones y velocidades de los astros. De otro, una tradición más antigua, que recoge Plinio, en la que el énfasis recae en las distancias, de modo que las diferencias en longitud entre los planetas se equiparan numéricamente a las producidas en las cuerdas de la lira heptacorde. Esta última tradición más antigua evoca, al referir la lira, los mentados experimentos de Pitágoras para establecer los números de los acordes consonantes. Destaquemos algo más, la novedosa denominación coelestis musica ${ }^{13}$. Honorio no desarrolla ningún tratamiento sobre esta nominación; no obstante, en los siglos XIII y XIV esta tendrá un alto rendimiento, siguiendo las premisas de Honorio.

El capítulo siguiente al de la música celestial se refiere al hombre. El hombre es un “microcosmos” (Migne, 1857-1866, CLXXII, col.1116). Este tópico, es abordado en varias obras de Honorio. Dada la homologación de la sustancia corpórea del hombre con los cuatro elementos, el hombre pasa a ser una versión pequeña del mundo mismo (minor mundus). En Elucidarium encontramos numerosas analogías: el cuerpo está hecho del elemento tierra; la sangre del agua; la respiración del aire; y el calor del fuego. Nuestras cabezas son redondas dado que reflejan las esferas celestes. Las siete aberturas de los órganos de los sentidos son como los siete tonos que ordenanornamentan al universo con armonía. Nuestra vista sale del fuego, oímos por el aire externo, y podemos sentir olores desde el aire interno al cuerpo; el agua nos da sabor y la tierra tacto (Migne,

\footnotetext{
11 También Boecio los ordena así. Diversa es la presentación de Platón: Tierra, Luna, Sol, Venus, Mercurio, Marte, Júpiter, Saturno y estrellas fijas; y Nicómaco: Tierra, Luna, Venus, Mercurio, Sol, Marte, Júpiter y Saturno. Este último autor, a su vez, otorga a la Luna el sonido más agudo, en oposición al resto de los autores.

12 T: tono, s/t: semitono. Cf. Plinio, Naturalis historia 2.19-20; Escoto Eirúgena, De divisione Naturae, PL, CXXII, col.722-723.

13 El Anónimo Barberini, atribuido por el editor J. Smits von Waesbergue a Adalboldo de Utrech, obispo entre $1010-1026$ refiere la voz musicae caelestis. Pero las agudas consideraciones de Ilnitchi (2002) obligan a posponer la datación para el siglo XIII.
} 
1857-1866, CLXXII, col.1116) ${ }^{14}$. La musica humana es un reflejo de la música de las esferas, es una consecuencia de la armonía entre los cuatro elementos y los poderes del alma.

La conformación del mundo mediante los cuatro elementos es tratada en De imagine: la tierra, elemento más pesado, ocupa la posición central en el sistema; el agua es más ligera y rodea la Tierra y la impregna; el aire llena el espacio entre la Tierra y la Luna (Flint, 1982, p.72); y el elemento más ligero, el fuego, se encuentra por sobre los demás. La esfera de aire (entre la Tierra y la Luna) era, según Honorio, habitada por demonios, cuyos cuerpos, como se les aparecía a las personas, estaban hechos de aire ${ }^{15}$. Entre estos elementos se producen una serie de transformaciones para dar lugar a las diferentes instanciaciones del mundo sublunar (Flint, 1982, p.50).

Ahora bien, lo que resulta una innovación de Honorio es la explicación acerca de por qué no escuchamos la música de las esferas, explicación que destella un grado de originalidad del autor. Recordemos que luego de la formulación platónica, Aristóteles en De caelo descartó la veracidad de la teoría de la música cósmica por la falta de comprobación empírica ${ }^{16}$. La totalidad de los autores subsiguientes no pudieron escapar a tales objeciones. Honorio propone una explicación. En De imagine establece que el hombre sólo puede oír sonidos producidos en el aire - como vimos, cada elemento se vincula a un sentido-; pero resulta que los sonidos de la armonía de las esferas no se producen en el aire, que sólo llena el espacio por debajo de la esfera de la Luna. Es esta la razón por la cual el sonido no nos alcanza y no podemos oírlo: "pues ese sonido no llega a nuestras orejas" (Flint, 1982, p.79) ${ }^{17}$, aunque resuena en todo el universo. La conformación de la esfera sublunar está dada por los cuatro elementos; más allá de ella domina el éter, tema que Honorio desarrolló en capítulos anteriores. En efecto, el espacio entre la Luna y la esfera celestial está compuesto de éter, elemento de "un alegre esplendor eterno, que los ángeles usaron para asumir sus cuerpos astrales cuando necesitaban ser visibles" (Flint, 1982, p. 75).

\footnotetext{
14 En De animae exsilio et patria los viajeros del alma son arrojados al exilio interior (el cuerpo) por su ignorancia y viajan a través de diez ciudades (artes liberales, física, mecánica, y economía) en su regreso a la sabiduría. En la quinta ciudad, la ciuitas musica, se les enseña que deben pasar por la melodía de la moral en el camino hacia la concordia- concierto celestial (Migne, 1857-1866, CLXXII, col.1244). Este estrecho vínculo entre música y ética, datado desde la Antigüedad, se basa en las concepciones metafísicas de la constitución musical de los cielos.

15 La tesis de que el aire alrededor de la Tierra está lleno de demonios ya estaba en Agustín, así como en Plutarco y Calcidio.

16 Reproducimos uno de los argumentos aristotélicos: “A algunos, en efecto, les parece forzoso que, al trasladarse cuerpos de semejante tamaño, se produzca algún sonido, ya que también < se produce $>$ con los próximos a nosotros, aun no teniendo el mismo tamaño ni desplazándose con una velocidad comparable: que, al desplazarse el sol y la luna, además de astros tan numerosos y grandes, en una traslación de semejante velocidad, es imposible que no se produzca un sonido de inconcebible magnitud. Suponiendo esto, así como que, en función de las distancias, las velocidades guardan

<entre sí> las proporciones de los acordes musicales, dicen que el sonido de los astros al trasladarse en círculo se hace armónico" (Candel, 1996, 290b15-23)

17 Qui sonus ideo ad nostras aures non pervenit.
} 
Si bien Honorio no explicita referencias en De imagine, podemos hipotetizarlas. En primer lugar, De caelo. Allí Aristóteles establece claramente la diferenciación de las regiones supra y sublunares y los elementos que las constituyen. (Claro que el Filósofo no incorpora las consecuencias que tendría su propia teorización del quinto elemento en relación con la crítica a la teoría de la música de las esferas). Si bien la obra aristotélica resulta disponible de manera completa para el Medioevo recién en el siglo XIII, con anterioridad ya circulaban ciertas traducciones. Entre las obras que los árabes recuperaron, destacan de la filosofía natural aristotélica: Física, De caelo, De anima, De generatione et corruptione, y Meteorológicos (Minecan, 2015, p. 32 y ss). Aun más, debemos considerar que estas conceptualizaciones aristotélicas estaban asumidas en autores que desarrollaron versiones de la música de las esferas. En efecto, el éter es nombrado como el elemento en el que los astros se desplazan en Cicerón — quien aborda con particular detenimiento la cuestión del éter en las menciones sobre el perdido De philosophia aristotélico $^{18}$ _, Nicómaco de Gerasa ${ }^{19}$, Arístides Quintiliano ${ }^{20}$, Boecio ${ }^{21}$. La teoría del quinto elemento como el constituyente del firmamento circulaba desde el siglo I a.C., y, reproducida por tantos autores, contaba con regular aceptación, aunque no siempre se dispusiera del texto aristotélico que la formuló.

De modo que Honorio realiza una articulación lógica de diferentes desarrollos. La posibilidad de audición tal como se presenta en Elucidarium y en De imagine se la vincula al medio en el que efectivamente se propagan los sonidos, el aire. Dado que las esferas se desplazan en otro medio, el aire que nos envuelve no llega a ser afectado para que se produzca la sensación correspondiente en el sentido auditivo. Con esta novedad, se presenta la primera solución al problema de la inaudibilidad de la música de los cielos que no culpa a la imperfección humana, sino que apunta a las circunstancias físicas que hacen a la producción del sonido.

\section{Conclusiones}

Hemos reconstruido los argumentos de Honorio en relación a la música de las esferas. Cómo esta es el modelo de la musica humana y la base para postular al hombre como microcosmos. La novedosa denominación de musica coelestis — basada en la musica mundana de Boecio a la vez que en la larga tradición pitagórico-platónica - hace total justica a los desarrollos de Honorio. Dado que la música de los cielos está más allá de la Luna, resuena en las zonas etéreas, de ahí la inaudibilidad para los

\footnotetext{
18 Cf. Sobre la naturaleza de los dioses I 13, 33, 38, 107; II, 15-16, 37-38, 42, 44, 95-96, 107; Cuestiones académicas I 7 , 26; y Disputaciones tusculanas I 10, 17, 22, 26-27, 65-66, 412.

19 Cf. Manual de harmónica $\mathrm{V}$.

20 Cf. Sobre la música, III.

21 Cf. La consolación de la filosofía III, m. 9; IV, m.1.
} 
mortales, que sólo pueden escuchar lo que se produce en la esfera sublunar. Los seres humanos, formados por la mezcla de los cuatro elementos (fuego, aire, agua y tierra) solo pueden percibir aquellas producciones conformadas a su vez por estos elementos, que son asimismo el soporte de transmisión de lo percibido, cada uno asociado a un sentido. De modo que, la radical separación entre mundo sublunar y supralunar — de raigambre aristotélica-, la sustancial heterogeneidad de estas dos zonas del mundo, constituye el fundamento de la falta de audición de la música cósmica.

Si bien Honorio ha sido reconocido por la crítica como un autor que se dedicó a realizar recopilaciones y sistematizaciones de los saberes previos - aspecto que efectivamente puede comprobarse en sus trabajos más importantes, De imagine, Elucidarium - entendemos que hemos hallado un elemento en el cual aparece su original inventiva: la postulación de esta musica coelestis y su vinculación al quinto elemento. De este modo, la revisión de los argumentos en torno a la teoría de las esferas, permite una reconsideración sobre las lecturas tradicionales acerca de este autor realizadas por numerosos estudiosos y especialistas. Sin embargo, debemos señalar que en cuanto a la coelestis musica será el siglo siguiente el que extraerá las consecuencias y el cabal rendimiento de lo postulado por Honorio, cuando Juan de Zamora y Jacobo de Liega produzcan la explícita distinción entre musica mundana y musica coelestis.

\section{Referencias bibliográficas}

CALABRESE, Claudio, DOMÍNGUEZ, Carlos (2015). Honorio de Autun. Comentario sobre el Timeo de Platón. Universidad Nacional de Mar del Plata: Mar del Plata.

CANDEL, Martín (1996). Aristóteles. Acerca del cielo. Gredos: Madrid.

COLOMER, Luis, GIL, Begoña (1996). Arístides Quintiliano. Sobre la música. Gredos: Madrid.

CROUSE, Robert (1975). “Honorius Augustodunensis: Disciple of Anselm?”, Analecta Anselmiana. Untersuchungen über Person und Werk Anselms von Canterbury, pp. 131-139.

ECO, Umberto (2013). Arte y belleza en la estética medieval. Sudamericana: Buenos Aires.

ESCOBAR, Ángel (1999). Cicerón. Sobre la naturaleza de los dioses. Gredos: Madrid.

FLINT, Valerie (1972). “The career of Honorius Augustodunensis. Some Fresh Evidence”, Revue Bénédictine, vol. 82, pp. 80-84. 
FLINT, Valerie (1977). "The place and purpose of the Works of Honorius Augustodunensis”, Revue Bénédictine, vol. 87, pp. 97-127.

FLINT, Valerie (1982). "Honorius Augustodunensis: Imago mundi." Edición. Archives d'histoire doctrinale et littéraire du Moyen Âge, vol. 49, pp. 7-153.

FRIEDLIN, Godofredus, MATHIESEN, Thomas ([1867]-1994). Boethius, Anicius Manlius Severinus. De institutione musica. Edición electrónica en Thesavrvs Mvsicarvm Latunarvm.

GARRIDO DOMENÉ, Fuensanta (2016). Los teóricos menores de la música griega. Euclides el geómetra, Nicómaco de Gerasa y Gaudencio el Filósofo. Cerix: Barcelona.

GARRIGUES, Marie (1986). L'oeuvre d'Honorius Augustodunensis: Inventaire critique. Verlag Erich Goltze KG: Göttingen.

GOTTSCHALL, David (1992). Das 'Elucidarium' des Honorius Augustodunensis: Untersuchungen zu seiner Überlieferung und Rezeptionsgeschiste im deutschprachigen Raum. Max Niemeyer Verlag:Tübingen.

HAHM, David (1982). "The fifth element in Aristotle's De Philosophia: a critical re-examination", Journal of Hellenic Studies, vol. 102, pp. 60-74.

ILNITCHI, Gabriela (2002). “'Musica Mundana', Aristotelian Natural Philosophy and Ptolemaic Astronomy", Early Music History, vol. 21, pp. 37-74.

KONIK, Marcin (2012). "A reception of the idea of the music of the spheres in the music theory of the twelfth and thirteenth centuries", Musica Iagellonica, pp. 5-50.

LUCENITINI, Paolo (1974). Honorius Augustodunensis. Clavis physicae. Edición. Éd. di Storia e letteratura: Roma.

LUFF, Robert (1999). Wissensvermittlung im Europaischen Mittelalter. Gruyter: Tübingen.

LUQUE MORENO, Jesús, LÓPEZ EISMAN, Antonio (2007). Agustín. Sobre la música. Gredos: Madrid.

LUQUE, Jesús, FUENTES, Francisco, LÓPEZ, Carlos, DÍAZ, Pedro, MADRID, Mariano (2009). Boecio. Sobre el fundamento de la música. Gredos: Madrid. 
MEDINA GONZÁLEZ, Alberto (2005). Cicerón. Disputaciones tusculanas. Gredos: Madrid.

MIGNE, Jean Paul (1857-1866). Patrologiae cursus completus. Series latina. Boethius, Anicius Manlius Severinus. De institutione musica. Edición. vol. LXIII. Cassiodorus Vivariensis Abbas. De Artibus ac Disciplinis Liberalium Litterarum. vol. LXX; Honorius Augustodunensis. Elucidarium Sive Dialogus De Summa Totius Christianae Theologiae; De Imagine Mundi Libri Tres vol. CLXXII. Garnier: Paris.

MINECAN, Ana (2015). "Recepción de la física de Aristóteles por Tomás de Aquino: Finitud, necesidad, vacío, unicidad del mundo y eternidad del universo". Tesis Doctoral, Facultad de Filosofía, Universidad Complutense de Madrid. Director: R. R. Guerrero.

PETERS, Francis (1968). Aristoteles Arabus. The Oriental translations and commentaries on the Aristotelian Corpus. Brill: Leiden.

ROTH, Alfred (1939). "Kleine Beiträge zur Kenntnis des sogenannten Honorius Augustodunensis”, Studia Neophilologica, vol. 12, pp. 120-135.

TATARKIEWICS, Wladysíaw (1989). Historia de la Estética. II La estética medieval. Akal: Madrid.

VILlALAZ, José (2011). Hugo de San Víctor. Didascalicon. Del arte de leer. Diecisite ed.: México. 